\section{DLG5 variants and susceptibility to inflammatory bowel disease in the Scottish population}

The recent study by Noble et al (Gut 2005;54:1416-20) did not find an association of the DLG5 G113A polymorphism with either inflammatory bowel disease (IBD) or Crohn's disease (CD). Previous studies in other European populations had reported an increase in 113A allele frequency in IBD and CD patients ${ }^{12}$ but, in striking contrast, Noble et al reported a trend towards decreased carrier frequency for the $113 \mathrm{~A}$ allele in IBD $(p=0.069)$ and $C D(p=0.057)$ patients. We believe there is reason to re-examine the genotype data used in the report of Noble et al.

Firstly, there was a significant difference in the proportion of missing genotypes between the IBD patients and controls $(\mathrm{p}<0.00001$, Fisher's exact test). Using the data in tables 1 and 3 , the missing genotype rate was $13 \%$ in controls and $4 \%$ in IBD patients. This difference strongly suggests differences in DNA quality or genotyping process between the IBD and control samples.

Secondly, using the data in table 3, the Gl13A polymorphism was not in HardyWeinberg equilibrium (HWE) in the IBD patients (HWE p value $=0.0056$, likelihood ratio test). HWE describes the relationship of allele and genotype frequencies in a randomly mating population, and departure from HWE in controls is commonly used to test for genotyping error. ${ }^{3}$ If the G113A polymorphism is independent of IBD disease status, then we expect the G113A polymorphism to be in HWE in IBD patients. There are fewer heterozygote IBD patients than expected under HWE, and this may be related to differences in heterozygote frequencies between IBD patients and controls $(\mathrm{p}=0.033)$ and between CD patients and controls $(p=0.029)$ reported by Noble et al. One possible explanation for this difference in heterozygote frequencies is genotyping error in IBD patients caused by allelic dropout of the 113A allele.

The difference in missing genotype rates and the departure from HWE in IBD patients suggest that the quality of the genotype data that underlie the conclusions of this study should be investigated.

B L Browning

Correspondence to: $\operatorname{Dr}$ B L Browning, Nutrigenomics Program, University of Auckland, Private Bag 92019 Auckland 1001, New Zealand b.browning@auckland.ac.nz

Conflict of interest: None declared.

\section{References}

1 Stoll M, Corneliussen B, Costello CM, et al. Genetic variation in DLG5 is associated with inflammatory bowel disease. Nat Genet 2005;36:476-80

2 Daly MJ, Pearce AV, Farwell L, et al. Association of DLG5 R30Q variant with inflammatory bowel disease. Eur J Hum Genet 2005; 13:835-9.

3 Leal SM. Detection of genotyping errors and pseudo-SNPs via deviations from HardyWeinberg equilibrium. Genet Epi 2005;29:204-14.

\section{Authors' reply}

We note Dr Browning's interest in our work. Unfortunately, we find his letter to be factually incorrect and therefore scientifically misleading.

In attempting to analyse further our own data, he has made invalid assumptions regarding our data set, which render his calculations inaccurate and his conclusions inappropriate. Moreover, the selected literature references which he quotes omit critical recent data and reviews of this area, and are not representative of the current state of knowledge.

In response to requests from the scientific referees of our paper, we included data on 294 controls who had been typed for the DLG variants, the OCTNl/2 variants, or both OCTN1/2 and DLG5 variants. The OCTNl/2 data (not the focus of this paper) were added as a marker independent of the DLG5 gene, in order to show that the two sets of controls were homogeneous. Of these 294 controls, DLG5 genotyping was undertaken in 269 samples, and successful genotypes obtained in 256 (95.2\%). Thus the failure rate in controls $(4.8 \%)$ was similar to that in IBD patients ( $4.4 \%, p=0.78$ by $\chi^{2}$ analysis). The HardyWeinberg data which Dr Browning derives are not attributable to genotyping failure.

The contribution of the DLG5 gene is clearly complex. Dr Browning fails to quote a series of large studies in adult European populations which are directly pertinent to our work. In the same volume of Gut as our paper, Torok and colleagues ${ }^{1}$ showed no association of the DLG5 113A variant in the German population with a non-significant reduction in 113A allelic frequency in Crohn' disease $(625$ Crohn's disease patients, 1012 controls). Vermeire et al performed studies in 2032 Belgian individuals, using strategies of case control analysis, as well as transmission disequilibrium testing, and noted significan undertransmission of the DLG5 113A allele towards affected offspring $\left(\mathrm{p}=0.01\right.$, by $\chi^{2}$ analysis. ${ }^{2}$ Data presented in abstract form from Cambridge ${ }^{3}$ and Oxford $^{4}$ showed no significant association (in the full paper, in press, final analysis of the Cambridge data again demonstrates non-significant reduction in 113A allelic frequency in Crohn's diseasepersonal communication, Miles Parkes).

It is noteworthy that unpublished data from our own unit suggest that in childhood onset disease, penetrance of the DLG5 113A allele may be critically influenced by sex and environmental influences (Russell et al, submitted).

These issues have recently been the subject of thoughtful editorials and correspondence, both in Gastroenterology, ${ }^{5}$ and most recently in the European Journal of Human Genetics. ${ }^{6}$ Unfortunately, these manuscripts were not considered by Dr Browning. We of course feel strongly that this area is clearly of great interest but we regretfully find the deficiencies in Dr Browning's letter to be potentially counterproductive.

J Satsangi, C Noble, I D R Arnott, R K Russell, E R Nimmo

Gastrointestinal Unit, Molecular Medicine Centre Western General Hospital, University of Edinburgh, Edinburgh, UK

Correspondence to: Professor J Satsangi, Gastrointestinal Unit, Molecular Medicine Centre, Western General Hospital, University of Edinburgh, Edinburgh EH4 2XU, UK; i.satsangi@ed.ac.uk Conflict of interest: None declared.

\section{References}

1 Torok H, Glas J, Tonenchi L, et al. Polymorphisms in the DLG5 and OCTN cation transporter genes in Crohn's disease. Gut 2005:54:1421-7.

2 Vermeire S, Pierik M, Hlavaty T, et al. Association of organic cation transporter risk haplotype with perianal penetrating Crohn's disease, but not with susceptibility to IBD. Gastroenterology 2005; 129:1845-53.
3 Waller S, Tremelling M, Bredin F, et al. Replication of association between IBD and TNF857, but not DLG5, NFKB, Keratin 8, or TUCAN/ CARD8. Gut 2005;54(suppl 2):A95

4 Cummings JRF, Herrlinger KR, Ahmad T, et al. Genotype-phenotype analyses of the IBD susceptibility gene DLG5. Gut 2005;54(suppl 2):A95

5 Trinh $\Pi$, Rioux JD. Understanding association and causality in the genetic studies of inflammatory bowel disease. Gastroenterology 2005; 129:2106-9.

6 Tenesa A, Noble C, Satsangi J, et al. Association of DLG5 and inflammatory bowel disease across populations. Eur J Hum Genet 2006; 14:259-60

7 Daly MJ, Rioux JD. Association of DLG5 and inflammatory bowel disease across populations. Eur J Hum Genet 2006;14:260-1.

\section{Dental erosion as extraoesophageal manifestation of gastro-oesophageal reflux}

Dental erosion can be considered as an extraoesophageal manifestation of gastrooesophageal reflux disease (GORD). Many reports have implicated gastric acid reflux as a contributory factor in the development of extraoesophageal disorders ("atypical" symptoms related to GORD), ${ }^{1}$ but the presence of lesions in the oral cavity in patients with acid reflux has been less studied. The association between acid reflux and dental erosion was first described by Howden in 1971 and was confirmed in other studies later, both in the adult population and in children.

We studied two groups of subjects: 181 patients with GORD and 72 healthy volunteers. ${ }^{2}$ Clinical assessment, including sex, age, body mass index, and consumption of tobacco and alcohol, was performed in all subjects, as well as a dental examination performed by a dental physician, blind to the diagnosis of the subjects. Parameters evaluated were the presence and number of dental erosions, location, and severity, according to the Eccles and Jenkins index, modified by Hattab. Clinical parameters were similar in both groups $(\mathrm{p}>0.05)$.

Compared with the control group, the percentage of dental erosions was significantly higher in the GORD group $(12.5 \% v$ $47.5 \%$; $<<0.001, \chi^{2}$ test), as was the number and severity of dental erosions $(\mathrm{p}<0.001$, Student's $t$ test). Location of dental erosions was significantly different between the groups. Given the high prevalence of dental erosions in patients with acid reflux, the collaboration between gastroenterologists and dentists is necessary in order to identify dental involvement in patients diagnosed with GORD. In contrast, subjects with unexplained dental erosions should be referred to gastroenterologists to investigate the presence of probable GORD.

A Benages, J V Muñoz, V Sanchiz, F Mora, M Mínguez

Department of Medicine, Hospital Clinic, University of Valencia, Valencia, Spain Professor A Benages, Department of Medicine, Hospital Clinic, University of Valencia, Valencia 46010, Spain; Benages@uv.es doi: 10.1136/gut.2006.093377

Conflict of interest: None declared.

\section{References}

1 Poelmans J, Tack J. Extraoesophageal manifestations of gastro-oesophageal reflux. Gut 2005;54:1492-9. 
2 Munoz JV, Herreros B, Sanchiz V, et al. Dental and periodontal lesions in patients with gastrooesophageal reflux disease. Dig Liver Dis 2003:35:461-7.

\section{Yoga for rehabilitation in chronic pancreatitis}

This study was conducted to determine the effectiveness of yoga in management of pain in patients with chronic pancreatitis. Thirty patients with chronic pancreatitis who were experiencing difficulties with pain, anxiety, and weight loss underwent a 12 week yoga programme. Twenty four patients completed the programme and comparison of the pain, weight, diet, and pain medication before and after the yoga programme was done. Findings suggest that yoga is effective in decreasing self perceptions of pain and anxiety in this population and reducing their usage of pain medication. Patients also had significant improvement in their diet and gained weight. Limitations of the study and recommendations for future research and practice are discussed.

The pain that develops in chronic pancreatitis can be severe, chronic, often aggravated by meals, and may be present continuously, including at night. At our centre, we offer a comprehensive approach to pain from pancreatic disease with clinical evaluation by a pain management specialist, pancreatic surgeon, gastroenterologist, and a psychologist, as indicated. There is a lack of prospective controlled studies comparing various treatment regimens. Thus treatment options are partly dependent on the experience of the physician taking care of the patient. ${ }^{1}$

Yoga was introduced as a tool for a rehabilitation programme and to supplement the other pain relief measures being taken by the patients. Yoga as a clinical intervention has been associated with a variety of physical and psychological health outcomes, such as improved mood and reduced symptoms of anxiety and depression. ${ }^{2}$ The yoga programme was designed to complement the management of pain and anxiety based on a sequence of yoga poses by BKS Iyengar. ${ }^{34}$

The yoga programme was implemented, directed, and evaluated by one of the authors (an experienced yoga instructor). Yoga sessions were planned three times a week over a 12 week period. Each session lasted approximately one hour. As participants entered the session, initial evaluation data were collected, including recording of each participant's pain levels, weight, average diet, and pain relief medication. Of 30 patients, 24 completed the full 12 week course.

Overall pre- and post-mean pain score was calculated according to the Von Korff pain score. ${ }^{5}$ The average pre-session pain score across all 36 sessions was 5.34. The average post-session pain score across all 16 sessions was 1.98 . On average, a $62 \%$ reduction in selfreported pain levels occurred for clients who participated in the yoga programme.

Average weight before the start of the yoga programme was $42.4 \mathrm{~kg}$, which increased gradually throughout the 12 week programme and the average weight after 12 weeks was $44.6 \mathrm{~kg}$ (that is, the average weight gained in 12 weeks was $5 \%$ ).

Prior to the start of the yoga intervention, participants received, on average, 2.64 as needed (PRN) pain medications each day; receipt of PRN pain medication was lower for all participants during the yoga intervention. This pattern of decreasing use of PRN pain medication was consistent for all participants, with data indicating that the average daily use of PRN pain medication decreased from 2.64 $(0.36)$ to $1.68(0.36)$. Paired Student's $t$ test was used for statistical calculations. SEM was 0.36 , with $\mathrm{n}=24$ and $\mathrm{p}<0.001$, which is highly significant. This represents a $36 \%$ reduction in participants' use of PRN pain medication during the yoga intervention.

Interviews were conducted with participants at the end of the 12 week yoga intervention to help determine its effectiveness. Each participant's thoughts regarding the yoga intervention were noted and all candidates claimed some benefit from the yoga programme. While each individual reported gains as a result of the yoga intervention, perhaps the best indicator of its effectiveness is found in the participants requests to continue the programme.

Because yoga is multifaceted, physical exercise, breathing, relaxation, and meditation is integrated into one's being when it is practiced. Such integration has a strengthening effect on the whole self, and fortifies resistance to pain. ${ }^{6}$ Yoga helps individuals become more accepting of their body and less judgmental and reactive to pain. ${ }^{7}$ Additionally, as individuals practice various yoga poses, their attention is gently directed to other parts of the body at any given moment.

The literature indicates that pain and anxiety are frequent and distressing Cooccurring disorders in patients with chronic pancreatitis that significantly detract from their health and well being. Based on this review, yoga may be a beneficial intervention to explore for managing pain and anxiety in clients with chronic pancreatitis.

S Sareen, V Kumari

Grantham and District Hospital, United Lincolnshire Hospitals NHS Trust, Grantham, UK

Correspondence to: Dr S Sareen, Grantham and District Hospital, United Lincolnshire Hospitals NHS Trust, 6 Norton St, Grantham NG31 6BY, UK surinder_sareen@yahoo.com doi: 10.1136/gut.2006.093088

Conflict of interest: None declared.

\section{References}

1 Moosner J. Chronic pancreatitis: nutrition and pain therapy. Schweiz Rundsch Med Prax 1998;87:1548-57.

2 Dattilo J. Facilitation techniques in therapeutic recreation. State College, Pennsylvania: Venture Publishing, 2003:112-14.

3 Pereira J. Living with AIDS through yoga and meditation. Yoga J 1995; 122:28-30

4 Purves AM, Penny K, Munro C, et al. Defining chronic pain for epidemiological researchassessing a subjective definition. Pain Clin 1998;10:39-147.

5 Von Korff M, Dworkin SF, Le Resche L. Graded chronic pain status: an epidemiologic evaluation. Pain 1990;40:279-91

6 Thompson AR, Wolfe JJ. Chronic pain management in the surgical patient. Surg Clin North Am 2005;85:209-24.

7 Sullivan MD, Turner JA, Romano J. Chronic pain in primary care: identification and management of psychosocial factors. J Fam Pract 1991;32:193-9.

8 Smith BH, Hopton JL Chambers WA. Chronic pain in primary care. Fam Pract 1999;16:475-82.

\section{Site distribution of gastrointestinal carcinoids differs} between races

We read with great interest the guideline for gastroenteropancreatic neuroendocrine (including carcinoid) tumours by Ramage et al (Gut 2005;54(suppl 4):ivl-16). In these excellent guidelines, they reported that the appendix is the most frequent site of gastrointestinal primary endocrine tumours (35\% of the total number of tumours) while colon and rectal carcinoids comprise only $7 \%$ and $10 \%$, respectively. One important aspect not mentioned however was the fact that site distribution of gastrointestinal carcinoids differs among races.

Modlin et al previously reported a marked predominance of carcinoids of the colon and appendix in the White Caucasian population based on nationwide surveillance in the USA. In contrast, the incidence of rectal carcinoids was 3-4-fold higher in African-Americans than in White Caucasians. Accordingly, the site distribution of colorectal carcinoids markedly differs among these two races.

Regarding carcinoids in the Asian population, there have been few reports based on a large database. In order to provide such evidence, we analysed colorectal carcinoids in the Japanese population using "the MultiInstitutional Registry of Large Bowel Cancers in Japan", a nationwide database which covers approximately $10 \%$ of the Japanese population, from 1984 to $1998 .{ }^{2}$ Among 90057 cases of colorectal tumours registered during this period, 345 cases of colorectal carcinoids were identified. All cases were in the Asian population. Site distribution was: the ileum, three cases $(0.9 \%)$; appendix, eight cases $(2.3 \%)$; colon, 28 cases $(8.2 \%)$; and rectum, 304 cases $(88.6 \%)$. Thus carcinoids in the Japanese population exhibited a much higher distribution in the rectum than in the colon or appendix. This overrepresentation of rectal carcinoids is compatible with a previous report from Taiwan in which $33(89.2 \%)$ of 37 colorectal carcinoids originated from the rectum. ${ }^{3}$

Differences in the distribution of colorectal carcinoids among races suggest that race related genetic factors play an important role in the development of gastrointestinal carcinoids. Hence racial disparity should be considered in the diagnosis of carcinoids, and further investigation using a larger database is needed to clarify these points.

T Konishi, T Watanabe Department of Surgical Oncology, University of Tokyo, Tokyo, Japan

T Muto

Department of Surgery, Cancer Institute Hospital, Tokyo, Japan

K Kotake

Department of Surgery, Tochigi Cancer Centre, Tochigi, Japan

H Nagawa

Department of Surgical Oncology, University of Tokyo, Tokyo, Japan

on behalf of the Japanese Society for Cancer of the Colon and Rectum

Correspondence to: Dr T Watanabe, Department of Surgical Oncology, University of Tokyo, 7-3-1 Hongo, Bunkyo-ku, Tokyo 113-8655, Japan; toshwatanabe@yahoo.co.jp

Conflict of interest: None declared.

\section{References}

1 Modlin IM, Lye KD, Kidd M. A 5-decade analysis of 13,715 carcinoid tumors. Cancer 2003;97:934-59.

2 Kotake K, Honjo S, Sugihara K, et al. Changes in colorectal cancer during a 20-year period: an extended report from the multi-institutional 